PID 20-21_126

\title{
ENOFOOD-GAME: Aplicación de la plataforma Kahoot como metodología activa de aprendizaje basado en la gamificación en estudios universitarios de grado y máster de la ETSIIAA de Palencia.
}

\author{
José Manuel Rodríguez Nogales 1, Encarnación Fernández Fernández 1, Josefina Vila Crespo 2 , \\ Violeta Ruipérez Prádanos 2, Raúl Moyano Gracia 2. \\ 1 Departamento de Ingeniería Agrícola y Forestal, 2 Departamento Anatomía patológica, Microbiología, Medicina preventiva y Salud pública, \\ Medicina legal y Forense \\ Email del coordinador: rjosem@iaf.uva.es
}

\begin{abstract}
RESUMEN: El proyecto ENOFOOD-GAME se planteó en el curso pasado, como una experiencia piloto para mejorar la motivación y el aprendizaje de los estudiantes empleando una herramienta de aprendizaje cooperativo y competitivo utilizando la plataforma digital Kahoot basada en la gamificación. Debido al alto grado de satisfacción de los estudiantes y profesores que participaron en la experiencia piloto, en este curso 20-21, esta herramienta de gamificación se reforzó en los títulos del curso pasado y se trasladó a otras dos asignaturas del Grado de Enología y a dos asignaturas del Máster en Calidad, Desarrollo e Innovación en Alimentos. Todos los estudiantes matriculados participaron en todas las sesiones y mostraron una satisfacción global muy alta con el uso de dicha plataforma, similar entre los estudiantes de grado y máster y entre los cursos 2019-20 y 2020-21. Los resultados obtenidos indican que la implementación de la plataforma digital Kahoot en asignaturas ha resultado ser una herramienta de mejora docente que ha mostrado unos resultados satisfactorios.
\end{abstract}

PALABRAS CLAVE: proyecto, innovación, docente, docencia, evaluación, continua, aprendizaje, colaborativo, gamificación

\section{INTRODUCCIÓN}

En el curso 19-20, el proyecto ENOFOOD-GAME se planteó como una experiencia piloto para mejorar la motivación y el aprendizaje de los estudiantes empleando una herramienta de aprendizaje cooperativo y competitivo que deriva del empleo de la gamificación, como es el uso de una plataforma digital Kahoot. Se propuso como una experiencia piloto para los Grados en Enología y en Ingeniería de las Industrias Agrarias y Alimentarias, y de los PEC I-ENOFOOD y I-AGRIFOOD, por lo que los primeros beneficiarios fueron los estudiantes matriculados en las asignaturas que participan en el programa. La satisfacción de los estudiantes y del profesorado con el PID ENOFOOD-GAME fue muy positiva. Los resultados de las encuestas mostraron que se ha mejorado la motivación, el aprendizaje y la evaluación de los estudiantes de los Grados en Enología y en Ingeniería de las Industrias Agrarias y Alimentarias y de los PEC I-ENOFOOD y I-AGRIFOOD por las materias que cursan empleando Kahoot como herramienta de gamificación. Además, los estudiantes y profesores manifestaron su interés en implementar la gamificación con Kahoot a otras asignaturas de estas y otras titulaciones.

En base a los resultados de las encuestas de satisfacción de los estudiantes y profesorado, y a las propuestas de mejora señaladas en el informe del PID del curso pasado, los objetivos propuestos para el curso académico 20-21, que se suman a los objetivos de la convocatoria 19-20, son los siguientes:

- Implementar la herramienta Kahoot en otras asignaturas y planes de estudio de la ETSIIAA de Palencia.

- Reforzar las acciones de retroalimentación en las sesiones Kahoot mediante el uso de debates como herramienta de enseñanza-aprendizaje.

En concreto, en este curso 20-21, esta herramienta de gamificación se reforzó en los citados títulos y se trasladó a otras dos asignaturas del Grado de Enología y a dos asignaturas del Máster en Calidad, Desarrollo e Innovación en Alimentos (Tabla 1).

\section{HERRAMIENTAS Y RECURSOS UTILIZADOS}

Para ello, en primer lugar, se analizaron y seleccionaron las competencias de aprendizaje de las asignaturas más adecuadas para ser adquiridas mediante el uso de la plataforma Kahoot. En el Anexo 1 se recogen estas competencias. Se desarrollaron y aplicaron las metodologías y estrategias de gamificación en la docencia de dichas asignaturas mediante el uso de la aplicación Kahoot. En la Tabla 2 se indica el número de sesiones para cada asignatura. Además, se abordó su uso como herramienta de autoevaluación para el estudiante y/o de evaluación para el profesor. Finalmente, se evaluó el grado de satisfacción de los estudiantes por el uso de la gamificación en las aulas mediante encuestas diseñadas para tal fin.

Tabla 1. Asignaturas y títulos de la ETSIIAA de Palencia con participación en el PID en las convocatorias 19-20 y 20-21. 


\begin{tabular}{|c|c|c|}
\hline Convocatoria & Títulos de la ETSIIAA de Palencia & Asignaturas \\
\hline \multirow[t]{2}{*}{ PID 19-20 } & $\begin{array}{l}\text { Grado en Enología } \\
\text { PEC I-ENOFOOD }\end{array}$ & $\begin{array}{l}\text { Tecnología enológica I } \\
\text { Bioquímica y microbiología enológicas II } \\
\text { Cultura vitivinícola }\end{array}$ \\
\hline & $\begin{array}{l}\text { Grado en Ingeniería de las Industrias Agrarias y Alimentarias } \\
\text { PEC I-ENOFOOD } \\
\text { PEC I-AGROFOOD }\end{array}$ & Biotecnología alimentaria \\
\hline \multirow[t]{3}{*}{ PID 20-21 } & $\begin{array}{l}\text { Grado en Enología } \\
\text { PEC I-ENOFOOD }\end{array}$ & $\begin{array}{l}\text { Tecnología enológica I } \\
\text { Bioquímica y microbiología enológicas II } \\
\text { Cultura vitivinícola } \\
\text { Tecnología enológica II } \\
\text { Biotecnología enológica }\end{array}$ \\
\hline & $\begin{array}{l}\text { Grado en Ingeniería de las Industrias Agrarias y Alimentarias } \\
\text { PEC I-ENOFOOD } \\
\text { PEC I-AGROFOOD }\end{array}$ & Biotecnología alimentaria \\
\hline & Máster en Calidad, Desarrollo e Innovación en Alimentos & $\begin{array}{l}\text { Propiedades físicas de los alimentos. Análisis } \\
\text { sensorial } \\
\text { Nuevas tendencias en enología }\end{array}$ \\
\hline
\end{tabular}

Tabla 2. Fecha de realización de la sesión de formación en Kahoot, número y fechas de las pruebas Kahoot realizadas en cada asignatura

\begin{tabular}{|l|c|c|}
\hline Asignaturas & $\begin{array}{c}\text { Fecha de la sesión de } \\
\text { formación en Kahoot }\end{array}$ & $\begin{array}{c}\text { Número de pruebas } \\
\text { Kahoot realizadas }\end{array}$ \\
\hline Tecnología enológica I & $05 / 03 / 2021$ & 4 \\
\hline Tecnología enológica II & $06 / 04 / 2021$ & 2 \\
\hline $\begin{array}{l}\text { Bioquímica y microbiología } \\
\text { enológicas II }\end{array}$ & $03 / 03 / 2021$ & 3 \\
\hline Biotecnología enológica & $16 / 11 / 2020$ & 2 \\
\hline Cultura vitivinícola & $22 / 02 / 2021$ & 1 \\
\hline Biotecnología alimentaria & $01 / 02 / 2021$ & 4 \\
\hline $\begin{array}{l}\text { Propiedades físicas de los } \\
\text { alimentos. Análisis sensorial }\end{array}$ & $4 / 02 / 2021$ & 1 \\
\hline Nuevas tendencias en enología & $19 / 03 / 2021$ & 1 \\
\hline
\end{tabular}

Antes de la primera sesión Kahoot los estudiantes recibieron una sesión formativa para el empleo de la herramienta (Anexo 2). En todas las sesiones las preguntas se formularon en formato test ("quiz") con cuatro opciones y con una respuesta correcta. Se dejó un tiempo variable en cada sesión (entre 60 y 120 segundos) para responder a cada pregunta utilizando la modalidad "player vs player" en la que cada alumno responde individualmente a las preguntas. Se priorizó el acierto de la pregunta, sin tener en cuenta la velocidad en dar las respuestas, para no tener en cuenta la competitividad entre estudiantes, que no era el objetivo final. Tras la respuesta a cada una de las preguntas se generó un debate entre los estudiantes y el profesor, y se resolvieron las dudas surgidas.

Las sesiones de aplicación de la plataforma Kahoot se utilizaron como herramientas de autoevaluación para el estudiante y/o de evaluación para el profesor. Por una parte, inmediatamente después de la respuesta a cada una de las preguntas, el debate generado entre estudiantes y profesor sirvió de autoevaluación para el estudiante, se explicaron el porqué de cada respuesta correcta y/o incorrecta en cada una de las preguntas. También se empleó como herramienta de evaluación, de manera que, los resultados obtenidos en las sesiones con Kahoot formaron parte de la evaluación en continuo de las asignaturas. Se priorizó el acierto de la pregunta, sin tener en cuenta la velocidad en dar las respuestas, para no tener en cuenta la competitividad entre estudiantes, que no era el objetivo final.

Finalmente, se evaluó el grado de satisfacción de los estudiantes por el uso de la plataforma Kahoot para la docencia, mediante una encuesta diseñada para tal fin, que fue común para todas las asignaturas en las que se implementó esta herramienta (Tabla 3). Se trataba de una encuesta anónima con 11 ítems, en cada uno de los cuales los estudiantes tenían que puntuar su grado de satisfacción del 0 (poco) al 10 (mucho), y se realizó de manera asincrónica a través de la plataforma Campus Virtual de la UVa. 


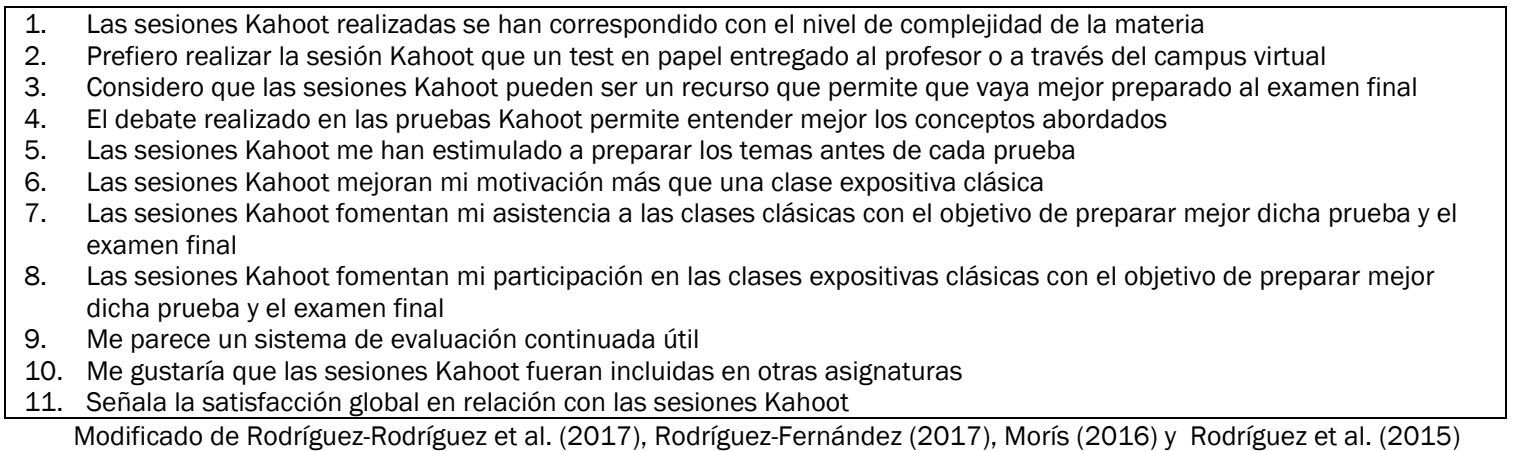

En la Figura 1 se recogen los resultados obtenidos para la encuesta de satisfacción a los estudiantes agrupados en estudios de grado y máster. Se han obtenido altas valoraciones para todos los ítems, no observándose diferencias estadísticamente significativas entre los estudios de grado y máster, a excepción de los ítems 2, 3 y 4 donde se alcanzó una puntuación mayor en los estudios de grado.

La satisfacción global en relación con las sesiones Kahoot (ítem 11) ha sido muy elevada, con valores de 8,7 y 8,2 para los estudios de grado y máster, respectivamente.

Respecto al ítem 2 (Prefiero realizar la sesión Kahoot que un test en papel entregado al profesor o a través del campus virtual) los alumnos de grado valoraron algo mejor esta pregunta $(7,7)$ que los de máster $(6,9)$. Además, los alumnos consideran que las sesiones Kahoot pueden ser un recurso muy útil que permite que vayan mejor preparado al examen final (ítem 3) (puntuaciones de 9,3 en grado y 8,1 en máster). Los estudiantes han valorado muy positivamente el debate que se realiza después de cada prueba Kahoot para mejorar la compresión de los conceptos abordados (ítem 4), con puntuaciones algo superiores para los estudios de grado $(9,2)$ que para los de máster $(8,2)$.

Hay que destacar las altas valoraciones dadas a la correspondencia entre las sesiones de Kahoot realizadas y el nivel de complejidad de la materia (ítem 1 con puntuaciones entre 8,4-8,7) y a la eficacia de las sesiones Kahoot para estimular al estudiante a preparar los temas antes de cada prueba (ítem 5 con puntuaciones entre 8,3-8,5). También se obtuvieron altas valoraciones para el ítem 7 (las sesiones Kahoot fomentan mi asistencia a las clases clásicas con el objetivo de preparar mejor dicha prueba y el examen final) con puntuaciones entre 7,8-8,1 y para el ítem 10 (me gustaría que las sesiones Kahoot fueran incluidas en otras asignaturas) con puntuaciones entre 8,0-8,5. Finalmente, los estudiantes valoraron positivamente las sesiones Kahoot en cuanto a que mejoran su motivación (ítem 6 con puntuación entre 8,2-8,3) y su participación en clase (ítem 8 con puntuaciones entre 6,8-7,7), y manifiestan que es una herramienta útil de evaluación (ítem 9 con puntuaciones entre 8,7-8,0)

Por otro lado, se compararon los resultados obtenidos en el curso 2019-20 y 2020-21 (Figura 2). En este caso no se observaron diferencias estadísticamente para ninguno de los 11 ítems analizados. La satisfacción global en relación con las sesiones Kahoot fue muy elevada, con puntuaciones de 8,4 y 8,5 para el curso 2019-20 y 2020-21, respectivamente.

Por otro lado, los profesores de las asignaturas consideran que la plataforma Kahoot ha resultado muy positiva como metodología de enseñanza-aprendizaje para la docencia en asignaturas universitarias, sin observarse diferencias estadísticamente significativas entre los cursos 2019-20 y 2020-21, a excepción del ítem 2. La satisfacción global con el uso de sesiones Kahoot fue elevada (ítem 1 con una puntuación de 9,0 y 9,6 en los cursos 2019-20 y 2020-21, respectivamente) (Tabla 4, Figura 3). Los profesores valoraron muy positivamente el uso de Kahoot para mejorar su motivación en comparación con una clase expositiva clásica (ítem 2 con puntuación de 10,0 y 9,0 en los cursos 2019-20 y 2020-21). Además, consideran que es una herramienta muy adecuada para la evaluación continua (ítem 3 con puntuaciones 8,8 y 9,2 en los cursos 201920 y 2020-21) y manifiestan que ampliarían el uso de Kahoot en otras asignaturas y/o recomendarían a otros compañeros que las incluyeran en otras asignaturas (ítem 4 con puntuaciones de 9,0 y 9,6 en los cursos 2019-20 y 2020-21). 


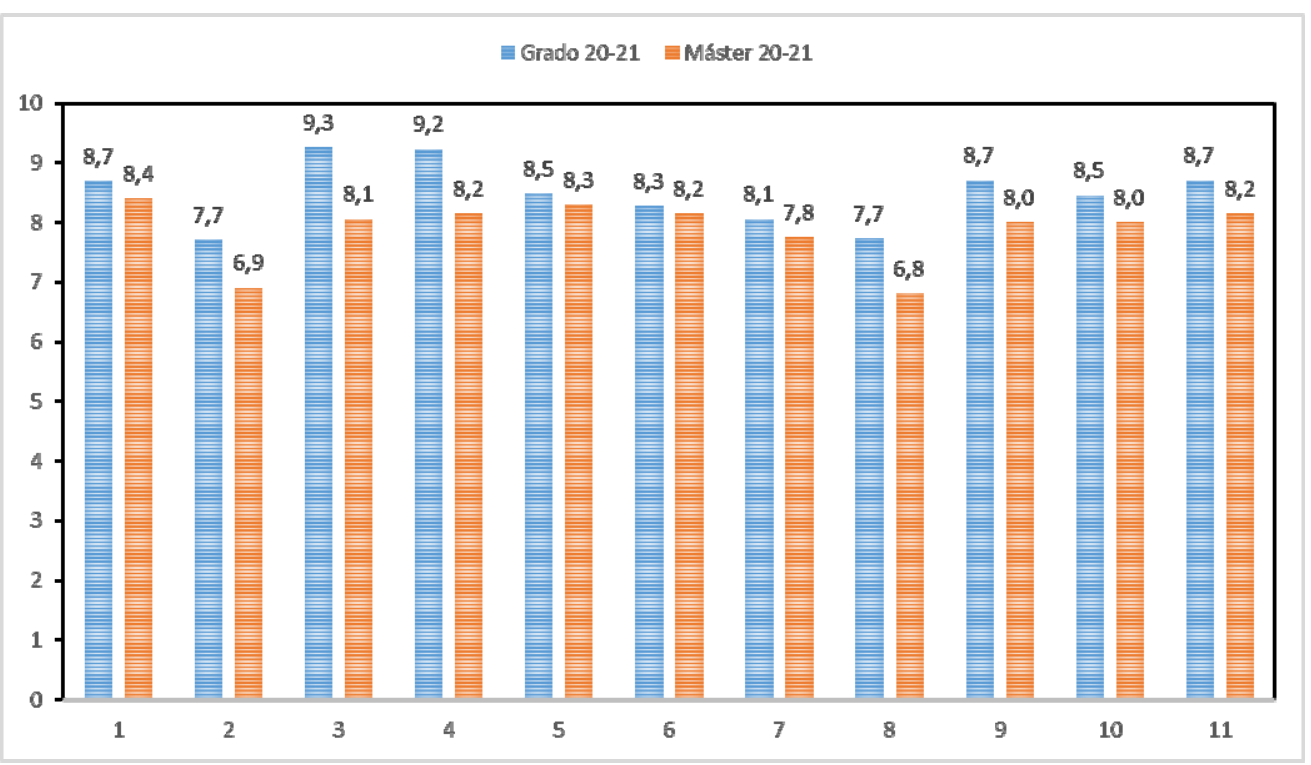

Fig. 1. Resultados de la encuesta de satisfacción de los alumnos de grado y máster para el curso 2020-21

플 2019-20 플 2020-21

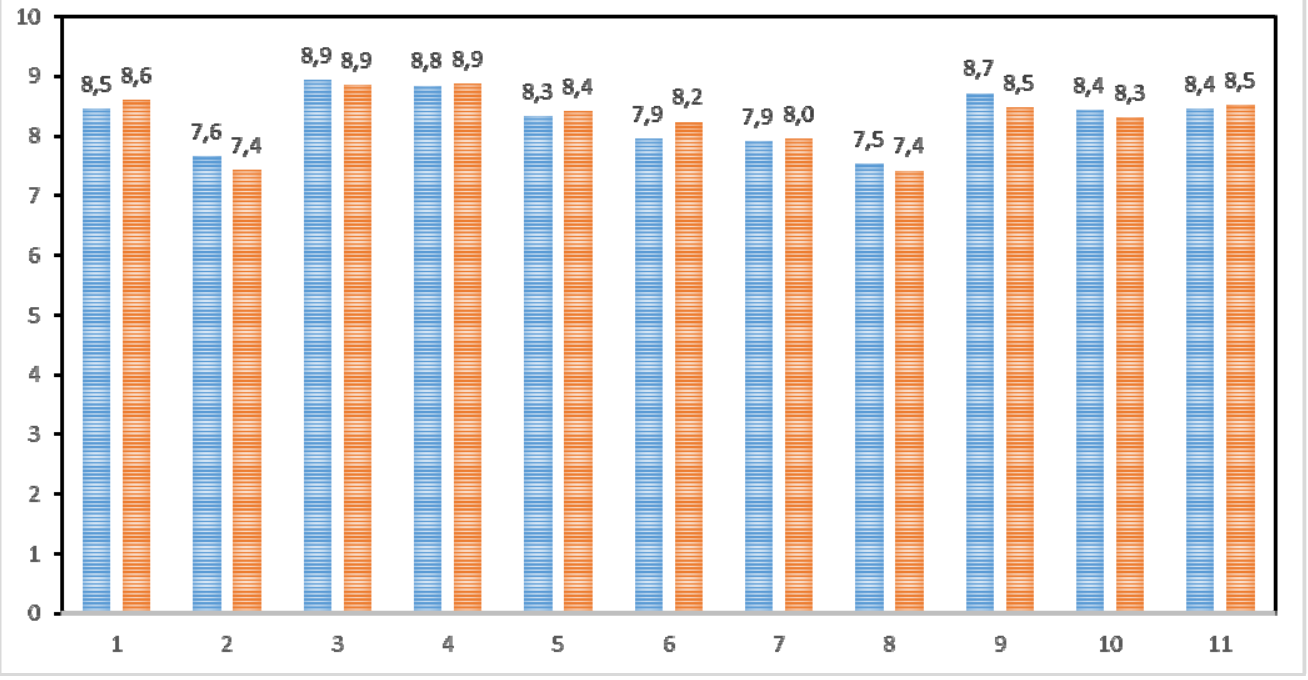

Fig. 2. Resultados de la encuesta de satisfacción de los alumnos para los cursos 2019-20 y 2020-21

En el anexo 3 se recogen las firmas de los alumnos que han participado en las sesiones Kahoot y en el anexo 4 se encuentran los informes individuales para cada asignatura donde se detalla la metodología de trabajo, los resultados obtenidos y el grado de cumplimiento de los objetivos. 
examen final?.

2. ¿La realización de sesiones Kahoot mejora tu motivación más que impartir una clase expositiva clásica?

3. ¿Te parece un sistema de evaluación continuada útil?

4. ¿Incluirías sesiones Kahoot en otras asignaturas y/o recomendarías a otros compañeros que las incluyeran en otras asignaturas?

5. Señala la satisfacción global en relación con las sesiones Kahoot.

Modificado de Rodríguez-Rodríguez et al. (2017), Rodríguez-Fernández (2017), Morís (2016) y Rodríguez et al. (2015)

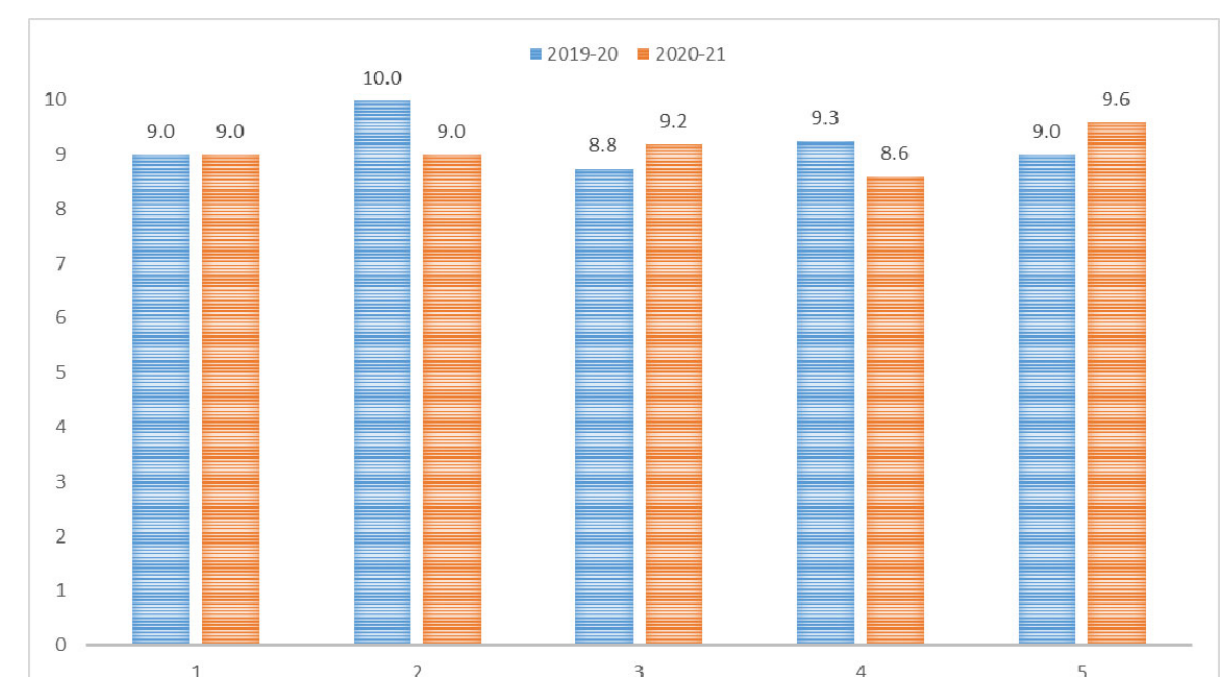

Fig. 3. Resultados de la encuesta de satisfacción de los profesores para los cursos 2019-20 y 2020-21

\section{GRADO DE CUMPLIMIENTO DE LOS OBJETIVOS Y PROPUESTAS DE MEJORA}

Los objetivos propuestos en las asignaturas se han cumplido de forma satisfactoria. En el anexo 5 se encuentra el grado de cumplimiento de las tareas del PID. La herramienta Kahoot ha sido recibida por los estudiantes con interés y ha demostrado ser útil para potenciar su motivación, facilitar el aprendizaje y la participación de los estudiantes.

Entre las dificultades que se han encontrado para la realización de las pruebas Kahoot en el aula destacan los problemas de conexión o escasa cobertura que hay en algunas zonas de la ETSIIAA. Debido a los protocolos COVID no ha sido posible realizar estas pruebas en el aula de informática y cada alumno ha llevado a cabo estas sesiones en su propio dispositivo móvil.

Por otro lado, los alumnos manifestaron la preferencia de los test en papel o en Campus Virtual durante la realización de las pruebas, explicando que la limitación de tiempo para responder cada pregunta era la causa de preferir otros métodos como sistema de evaluación. Sin embargo, destacaron su aplicación como herramienta destinada a fomentar la motivación y comprender y preparar el contenido abordado en la asignatura.

Se pretende continuar con el empleo de esta metodología, ya sea utilizando la plataforma Kahoot u otras similares como Socrative o Mentimeter, en los próximos años. Como puntos fuertes, destaca la alta aceptación de esta metodología por el alumnado, así como su empleo para mejorar la docencia ya sea presencial o virtual, como método de evaluación continua y como procedimiento para generar debate entre estudiantes y el profesorado, y su uso para entender mejor los conceptos de la asignatura.

Se han identificado aspectos que pueden ser mejorables en cursos futuros. En primer lugar, Kahoot ofrece diferentes modalidades para el desarrollo de sus cuestionarios. Se ha comenzado por el empleo más básico de la herramienta (quiz, individual), sin embargo, la competitividad e interés que ha suscitado su empleo apunta a que el empleo de este y otros nuevos formatos (verdadero/falso, open-ended, en equipo...) podrían ser utilizados con más frecuencia durante las clases para afianzar conceptos y generar debates que fomenten su interés y participación. Asimismo, se abre la posibilidad del empleo de nuevas herramientas o plataformas digitales (Socrative o Mentimeter) basadas en la gamificación como metodologías activas de aprendizaje.

\section{CONCLUSIONES}

El empleo de Kahoot como tecnología educativa innovadora en estudios de grado y máster ha permitido globalmente mejorar la motivación y el aprendizaje de los estudiantes, y de manera específica: 
- Incluir de manera satisfactoria el cuestionario kahoot como sistema de evaluación continua, por la facilidad de utilización en el aula y con buena acogida por el alumnado.

- Facilitar la adquisición y comprensión de los conceptos impartidos en la asignatura.

- Fomentar la participación activa de los alumnos y genera debates enriquecedores y dinámicos.

- La satisfacción global de los alumnos de grado y máster con la herramienta es similar y también entre los cursos 2019-20 y 2020-1.

- La satisfacción global de los profesores con Kahoot es similar entre ambos cursos académicos.

En conjunto, la implementación de Kahoot en la docencia de estas asignaturas ha sido bien aceptada y valorada tanto por los alumnos como por los profesores y ha resultado ser una herramienta de mejora docente que ha mostrado unos resultados satisfactorios.

\section{DIFUSIÓN DE LOS RESULTADOS}

Los resultados del PID se han presentado al III Congreso Internacional de Innovación Docente e Investigación en Educación Superior: Cambios en el proceso de enseñanza-aprendizaje de las Áreas del Conocimiento. 17, 18 y 19 de noviembre de 2021.

Título: "ENOFOOD-GAME: Aplicación de la gamificación en estudios universitarios de grado y máster del ámbito alimentario". José Manuel Rodríguez Nogales, Encarnación Fernández Fernández, Josefina Vila Crespo, Violeta Ruipérez Prádanos, Raúl Moyano Gracia. Grupo ENOBIOTEC. Universidad de Valladolid.

\section{ANEXOS}

PID_20_21_126_Anexo 1.pdf(listado de competencias a desarrollar con Kahoot)

PID_20_21_126_Anexo 2.pdf (listado de firmas de los estudiantes en la sesión de formación en Kahoot)

PID_20_21_126_Anexo 3.pdf (listado de firmas de los estudiantes en las sesiones Kahoot)

PID_20_21_126_Anexo 4.pdf (informes individualizados para cada asignatura)

PID_20_21_126_Anexo 5.pdf (grado de cumplimento de las tareas del PID)

PID_20_21_126_Anexo 6.pdf (carta de aceptación en el congreso III CIDICO)

Los anexos se encuentran alojados en: https://uvadoc.uva.es/handle/10324/47489

\section{REFERENCIAS}

1. Moris, G. (2016). Gamificación a través de Kahoot como innovación docente en el Grado de Logopedia. Convocatoria de los Proyectos de Innovación 2016. Universidad de Oviedo. http://www. innova. uniovi. es/c/document library/get file. [consultado el 15/02/2020].

2. Rodríguez, E., Aparicio, A., López, A. M., Ortega, R. M., Navia, B., Andrés, P., \& Cuadrado, J. E. (2017). Herramienta Kahoot como estrategia para fomentar la participación y el aprendizaje activo del alumno. Proyecto de Innovación y Mejora de la Calidad Docente 2016/2017. Universidad Complutense de Madrid. https://eprints.ucm.es/45131/1/Memoria\%20proyecto\%2043.pdf [consultado el 17/01/2020].

3. Rodríguez, F., Loro, F., \& Villén, S. (2015). Experiencia de gamificación en alumnos de magisterio para la evaluación de la asignatura Sociología de la Educación mediante el uso de la plataforma Kahoot. In $3^{\text {rd }}$ International Conference on Innovation, Documentation and Teaching Technologies (p. 223). http://ddfv.ufv.es/handle/10641/1268 [consultado el 17/02/2020].

4. Rodríguez-Fernández, L. (2017). Smartphones y aprendizaje: el uso de Kahoot en el aula universitaria. Revista Mediterránea de Comunicación/Mediterranean Journal of Communication, 8(1), 181-189.

\section{AGRADECIMIENTOS}

Los miembros del PID quieren agradecer a los estudiantes del Grado en Enología, Grado en Ingeniería de las Industrias Agrarias y Alimentarias, PEC I-ENOFOOD, PEC I-AGRIFOOD y del Máster de Calidad, Desarrollo e Innovación en alimentos por su participación en las sesiones Kahoot y en las encuestas de satisfacción. 